\title{
Assessment of the Electrocardiogram T-Wave Intervals with Acute Biliary Pancreatitis
}

\author{
Abdullah Algın, ${ }^{1}$ ๑ Hüseyin Avni Fındıklı, ${ }^{2}$ ๑ Burcu Genç Yavuz, ${ }^{3}$ ๑ Davut Tekyol, ${ }^{3}$ \\ Nihat Müjdat Hökenek, ${ }^{4}$ [D Avni Uygar Seyhan ${ }^{4}$
}

${ }^{1}$ Department of Emergency Medicine, University of Health Sciences Umraniye Training and Research Hospital, Istanbul, Turkey ${ }^{2}$ Department of Internal Medicine, Necip Fazıl City Hospital, Kahramanmaras, Turkey

${ }^{3}$ Department of Emergency Medicine, University of Health Sciences Hamidiye Faculty of Medicine, Haydarpasa Numune Health Application and Research Center, Istanbul, Turkey

${ }^{4}$ Department of Emergency Medicine, University of Health Sciences Kartal Dr. Lütfi Kırdar Training and Research Hospital, Istanbul, Turkey

\begin{abstract}
Introduction: Cardiovascular involvement is one of the multisystemic manifestations of acute biliary pancreatitis. In this study, we aimed to examine, to our knowledge, for the first time, how the Tpeak-Tend (TpTe) interval, a highly predictive electrocardiographic marker, is affected in patients with acute biliary pancreatitis

Methods: A total of 68 subjects were recruited in this retrospective case-control study. To determine the Corrected QT Intervals (QTC) and TpTe intervals, the electrocardiographs of all subjects were manually examined using the precordial V5 lead. Results: We found that the TpTe and QTc intervals were longer in the cases compared to the controls, and this difference was statistically significant $(p<0.05)$. With respect to acute biliary pancreatitis, the QTc had $68.4 \%$ sensitivity and $67 \%$ specificity (AUC=0.660, Cl: $0.529-0.791 ; p=0.024)$ and the TpTe interval had $76.3 \%$ sensitivity and $63.3 \%$ specificity (AUC=0.647, Cl: $0.508-$ $0.787 ; \mathrm{p}=0.038)$.

Discussion and Conclusion: The Tpeak-Tend interval was evaluated for the risk of arrhythmia in patients with acute biliary pancreatitis. Our study demonstrated how the Tpeak-Tend and OTc intervals were affected in patients with acute biliary pancreatitis and that acute pancreatitis might be one of the causes of prolonged QTc and Tpeak-Tend intervals.

Keywords: Acute pancreatitis; electrocardiogram; T-wave intervals.
\end{abstract}

A cute pancreatitis (AP) is an acute inflammatory condition in which activated proteolytic enzymes secreted by pancreatic acinar cells due to the action of various stimuli cause the pancreas to digest itself ${ }^{[1]}$. AP is manifested by abdominal pain and elevated serum pancreatic enzymes. AP is associated with variable involvement not only of pancreatic tissues but also of other organs. Although there are many causes of $A P$, the cause in more than $80 \%$ of cases is gallstone migration and alcohol consumption ${ }^{[2-4]}$.

In $\mathrm{AP}$, cardiovascular and/or pulmonary manifestations are common in multiple organ system dysfunctions. Cardiac manifestations of AP include vasomotor disorders and arrhythmias ${ }^{[5,6]}$. Sudden cardiac deaths, among the consequences of arrhythmias, cause approximately 800.000 deaths worldwide every year ${ }^{[7]}$. To our knowledge, studies investigating the risk of arrhythmogenicity in acute biliary pancreatitis (ABP) included QTc measurement ${ }^{[8,9]}$. We wanted to demonstrate how the TpTe interval, which in recent years has been used as a new and highly predictive marker for the risk of arrhythmia and cardiovascular death, is affected in ABP patients ${ }^{[10,11]}$.

Correspondence (iletişim): Abdullah Algın, M.D. Acil Tip Klinigi, SBU Umraniye Egitim ve Arastirma Hastanesi, Istanbul, Turkey Phone (Telefon): +90 5542763968 E-mail (E-posta): dralgin@hotmail.com 


\section{Materials and Methods}

This retrospective case-control study included a total of 68 individuals, including 38 cases and 30 controls. The control group was selected from age- and gender-matched patients. Due to both groups were formed using the archive database search, written informed consent was not obtained from patients. Ethical approval was obtained from the local ethics committee to conduct this study (ID: 2019/514/155/1).

This study included patients whose ABP diagnosis was established in the emergency department on the basis of acute abdominal pain and/or tenderness, using the laboratory data (elevated serum amylase, lipase, conjugated bilirubin, transaminase concentrations, and alkaline phosphatases), abdominal ultrasonography and/or computed tomography ${ }^{[12]}$. Patients whose data were missing or not available in the archive were excluded from this study.

They comprised individuals who had no known chronic disease and no history of drug-use known to affect the QT interval (e.g., antiarrhythmic drugs) and whose electrolytes $(\mathrm{Na}, \mathrm{K}, \mathrm{Ca})$, urea and creatinine levels were within the reference range of our biochemistry laboratory.

The cases with the resting electrocardiogram (ECG) (Nihon Kohden ECG 1250K) recorded at a paper speed of $50 \mathrm{~mm} /$ sec were included, and the ECGs were examined manually. The TpTe interval refers to the time between the peak and the end of the $T$ wave and was measured using the tangent method. The QT interval was measured as the time from the start of the QRS complex to the end of the T wave. The QTc interval was measured using Bazett's formula ${ }^{[13]}$. A precordial V5 lead was used for both measurements.

\section{Statistical Analysis}

Statistical analysis was performed using SPSS statistical software (version 21.0, SPSS, Inc., Chicago, IL). The normality of data was analyzed using visual plots (histograms and probability graphs), and the test of normality (the Kolmogorov-Smirnov test). The homogeneity of variance was analyzed using Levene's test. The variables with normal distribution in the descriptive analysis were expressed as mean \pm standard deviation (SD) values. The groups were compared concerning continuous variables using Student's t-test and about categorical variables using a chisquare test. Estimation values and predictions of independent predictors were analyzed with the receiver operating characteristic (ROC) curve. The ROC curve and the area under the ROC curve (AUC) were assessed using the method of Hanley and McNeil. An AUC close to 1.0 was interpreted as an elevated predictive value. The total type I error rate (significance level) was set to $5 \%$ in all statistical analyses.

\section{Results}

Our study groups were similar in age and gender $(p>0.05)$ (Table 1). The mean QTc (ms) and TpTe (ms) intervals were different between the groups. The TpTe interval was $88.13 \pm 10.1$ $\mathrm{ms}$ in the cases and $81.97 \pm 11.4 \mathrm{~ms}$ in the controls. The QTc was $423.8 \pm 7.72 \mathrm{~ms}$ in the cases and $420.1 \pm 5.74 \mathrm{~ms}$ in the controls. The TpTe and QTc intervals were longer in the cases compared to the controls, and this difference was statistically significant $(p<0.05)$ (Table 1$)$.

The comparison of Tp-e (Tpeak-Tend) and QTc (corrected QT) interval between the study groups is as in Figure 1. ROC

Table 1. Demographic and biochemical characteristics of the controls and the patients groups

\begin{tabular}{lccc}
\hline Variables Total & $\begin{array}{c}\text { Controls } \\
(\mathbf{n = 3 0 )}\end{array}$ & $\begin{array}{c}\text { Patients } \\
(\mathbf{n = 3 8 )}\end{array}$ & $\mathbf{p}$ \\
\hline Sex (male), \% & $11(36.7)$ & $8(21.1)$ & 0.154 \\
Age (years) & $56.7 \pm 16.3$ & $59.26 \pm 17.5$ & 0.540 \\
QTc Interval (ms) & $420.1 \pm 5.74$ & $423.8 \pm 7.72$ & $0.034^{*}$ \\
Tp-e Interval (ms) & $81.97 \pm 11.4$ & $88.13 \pm 10.1$ & $0.022^{*}$ \\
\hline
\end{tabular}

Values are presented as mean \pm standard deviation; QTc: Bezzet's correction; Tp-e: Tpeak and Tend interval.

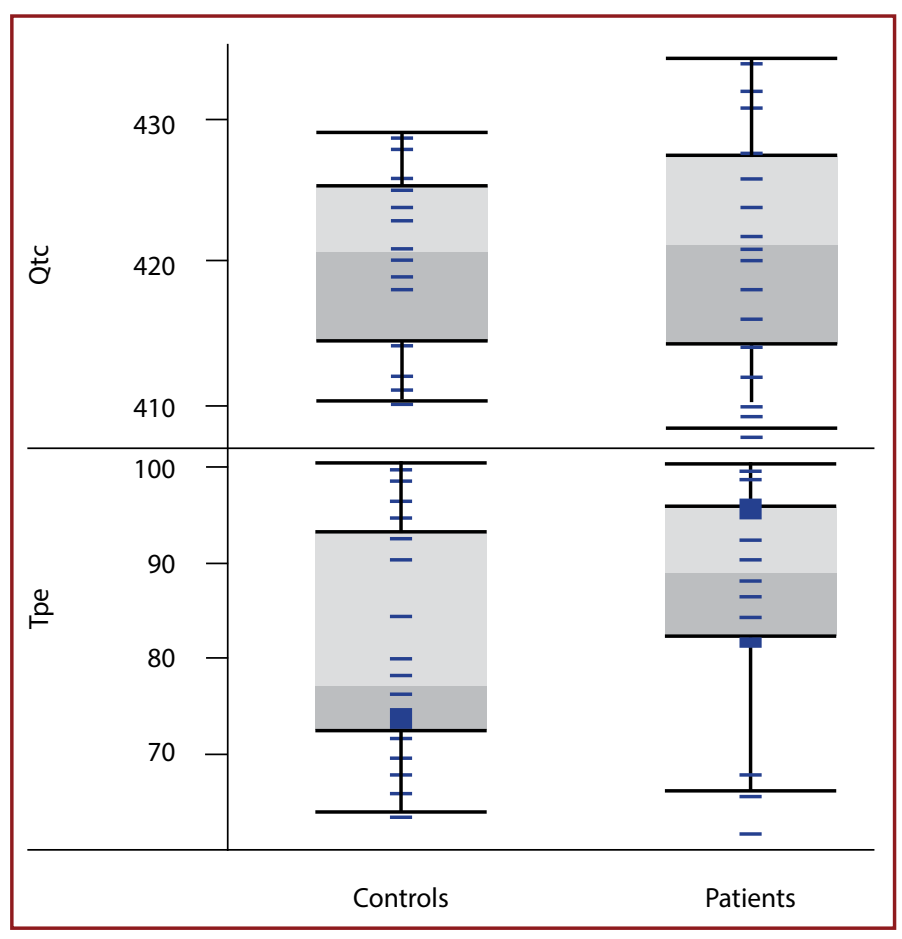

Figure 1. Comparison of Tp-e (Tpeak-Tend) and QTc (corrected QT) interval between the study groups. 
curves derived from electrocardiographic parameters were analyzed for the affectability of the TpTe interval and QTC by ABP. Accordingly, the QTc had $68.4 \%$ sensitivity and $67 \%$ specificity ( $A U C=0.660, \mathrm{Cl}: 0.529-0.791 ; \mathrm{p}=0.024$ ) with an optimal cut-off value of $421.5 \mathrm{~ms}$. The TpTe interval had $76.3 \%$ sensitivity and $63.3 \%$ specificity (AUC $=0.647, \mathrm{Cl}$ : 0.508-0.787; $\mathrm{p}=0.038$ ) with an optimal cut-off value of $85 \mathrm{~ms}$ (Fig. 2). The results of the TpTe interval were similar to the results of the QTc interval in sensitivity, specificity, and predictive values.

\section{Discussion}

Studies to date on electrocardiographic and arrhythmogenic markers have highlighted that the TpTe interval may be affected by various clinical entities in individuals without underlying heart disease and may be a predictive parameter for ventricular arrhythmias ${ }^{[14-16]}$. To our knowledge, for the first time in the literature, we investigated how the TpTe interval, as a new and highly predictive index of arrhythmogenicity, changes in pancreatitis patients. The main finding of our study was that the TpTe and QTc intervals were longer in the cases compared to the controls. Prolonged TpTe and QTc intervals, along with prolonged ventricular repolarization, indicate the risk of arrhythmia in pancreatitis patients.

It is not yet clear whether acute pancreatitis affects the TpTe interval. A literature search revealed that the clinical significance of the TpTe interval in patients with acute pancreatitis has not yet been studied. Considering studies to date on acute pancreatitis and electrocardiographic variables, Ates et al. ${ }^{[8]}$ studied patients with biliary pancreatitis and found that the QTc interval was longer in the cases compared to the controls. In another study, Sakagami et al. ${ }^{[17]}$ reported a prolonged QTc interval in patients with chronic alcoholic pancreatitis. In their study on a group of fifty-one patients, Tapia et al. ${ }^{[9]}$ found abnormal ECG findings in twentyeight patients (55\%). Consistent with earlier observations, this study also observed a difference in ECG between the groups (prolonged TpTe and QTc intervals in the cases). No published data has been found that measures the diagnostic value of electrocardiographic parameters and how electrocardiographic parameters are affected. We found that the QTc and TpTe intervals were similarly affected in ABP patients, and both parameters were diagnostic with greater than 421.5 and $85 \mathrm{~ms}$.

Several theories have been proposed to explain the pathogenesis of electrocardiographic changes during acute pancreatitises, such as electrolytes disturbances, direct injury to myocyte membrane due to the cardiotoxic effects of proteolytic enzymes, coagulopathy, cardiobiliary reflex, and coronary artery spasm ${ }^{[5]}$. Our patient group was selected from individuals who did not have a coagulopathy and electrolyte disorders at arrival to the emergency department. As there was no patient with biliary pancreatitis, a cardiobiliary reflex was unlikely. We observed no ST elevation indicative of a coronary artery spasm. Therefore, we believe that the cardiotoxic effects of proteolytic enzymes that arise from pancreatitis may be the cause of prolonged TpTe and QTc intervals in our study.

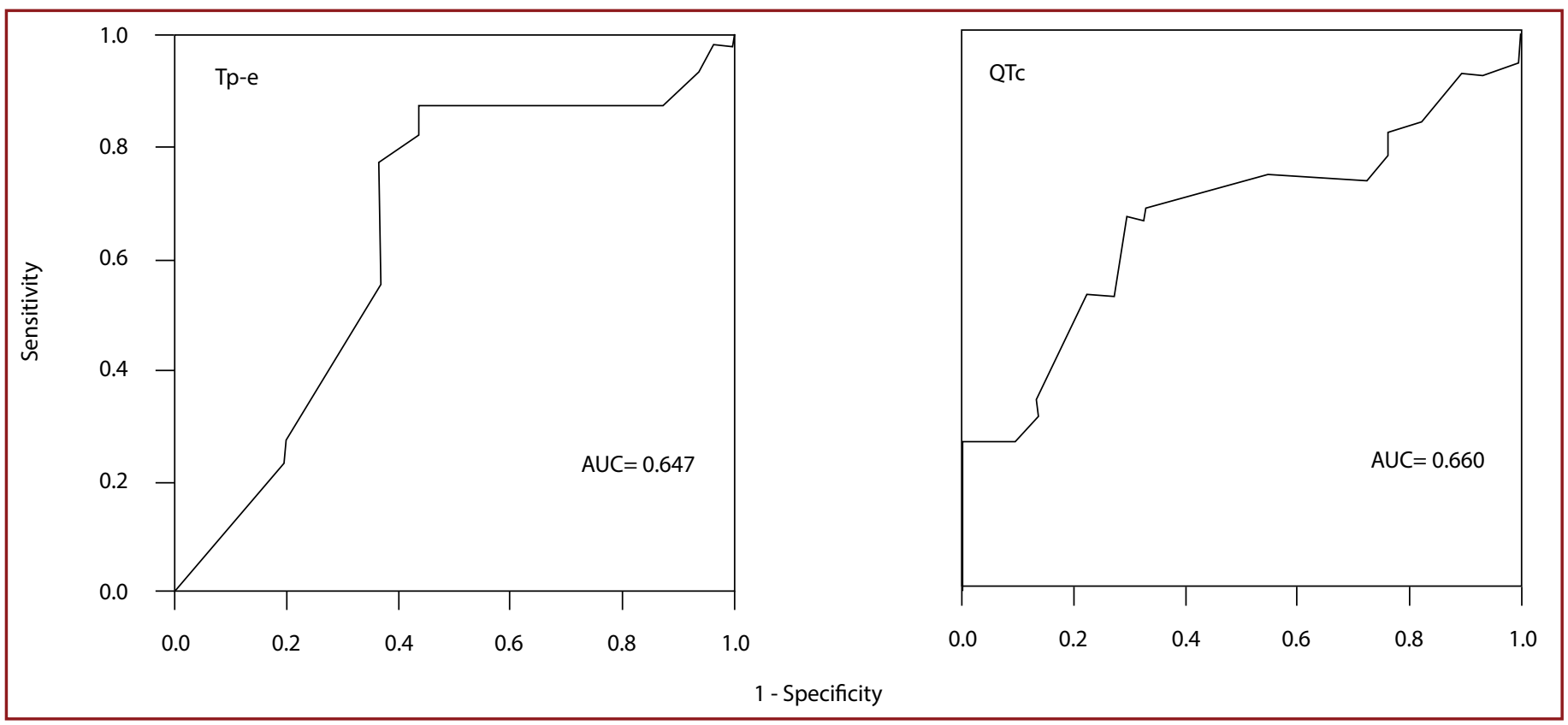

Figure 2. Performance of variables in predicting acute biliary pancreatitis stage. 
The major limitation of this study is perhaps that we were unable to provide information on the effect of disease severity on TpTe and QTc intervals due to the lack of records of early prognostic and disease severity index in ABP patients. Concerning further homogenization of TpTe and QTC intervals, we were also unable to compare the ECG of the same individual taken at admission to the emergency department and at discharge from the hospital (e.g., before and after). Other limitations are that this study was singlecentered and cross-sectional and included a small number of patients.

\section{Conclusion}

In conclusion, this study showed how the QTC and TpTe intervals were affected in $A B P$ patients and that $A B P$ might be one of the causes of prolonged QTc and TpTe intervals. Prospective randomized studies with a large sample size are needed to elucidate the association between regional and transmyocardial repolarization parameters and cardiac events.

Ethics Committee Approval: The present study was conducted according to the declaration of Helsinki and Ethics committee approval was obtained the study (ID: 2019/514/155/1).

Peer-review: Externally peer-reviewed.

Authorship Contributions: Concept: A.A., H.A.F.; Design: A.A., D.T.; Supervision: A.A., N.M.H.; Materials: A.A.; Data collection \&/ or processing: A.A.; Analysis and/or interpretation: N.M.H., D.T., B.G.Y.; Literature search: A.A., H.A.F.; Writing: A.A.; Critical review: A.A., B.G.Y., A.U.S.

Conflict of Interest: None declared.

Financial Disclosure: The authors declared that this study received no financial support.

\section{References}

1. Portelli $M$, Jones CD. Severe acute pancreatitis: pathogenesis, diagnosis and surgical management. Hepatobiliary Pancreat Dis Int 2017;16:155-9. [CrossRef]

2. Halangk W, Lerch MM. Early events in acute pancreatitis. Gastroenterol Clin North Am 2004;33:717-31. [CrossRef]

3. Carroll JK, Herrick B, Gipson T, Lee SP. Acute pancreatitis: diagnosis, prognosis, and treatment. Am Fam Physician 2007;75:1513-20.
4. Marx C. Adrenocortical insufficiency: an early step in the pathogenesis of severe acute pancreatitis and development of necrosis? Do we have a new treatment option? Crit Care Med 2006;34:1269-70. [CrossRef]

5. Yegneswaran B, Kostis JB, Pitchumoni CS. Cardiovascular manifestations of acute pancreatitis. J Crit Care 2011;26:225. e11-8. [CrossRef]

6. Agarwal N, Pitchumoni CS. Acute pancreatitis: a multisystem disease. Gastroenterologist 1993;1:115-28.

7. Zaidi M, Robert A, Fesler R, Derwael C, Brohet C. Dispersion of ventricular repolarisation: a marker of ventricular arrhythmias in patients with previous myocardial infarction. Heart 1997;78:371-5. [CrossRef]

8. Ates F, Kosar F, Aksoy Y, Yildirim B, Sahin I, Hilmioglu F. QT interval analysis in patients with acute biliary pancreatitis. Pancreas 2005;31:238-41. [CrossRef]

9. Rubio-Tapia A, García-Leiva J, Asensio-Lafuente E, RoblesDíaz G, Vargas-Vorácková F. Electrocardiographic abnormalities in patients with acute pancreatitis. J Clin Gastroenterol 2005;39:815-8. [CrossRef]

10. Yamaguchi $M$, Shimizu $M$, Ino $H$, Terai $H$, Uchiyama $K$, Oe $K$, et al. T wave peak-to-end interval and QT dispersion in acquired long QT syndrome: a new index for arrhythmogenicity. Clin Sci (Lond) 2003;105:671-6. [CrossRef]

11. Yan GX, Antzelevitch C. Cellular basis for the normal T wave and the electrocardiographic manifestations of the long-QT syndrome. Circulation 1998;98:1928-36. [CrossRef]

12. Ranson JH. Diagnostic standards for acute pancreatitis. World J Surg 1997;21:136-42. [CrossRef]

13. Funck-Brentano C, Jaillon P. Rate-corrected QT interval: techniques and limitations. Am J Cardiol 1993;72:17B-22B. [CrossRef]

14. Yontar OC, Karaagac K, Tenekecioglu E, Tutuncu A, Demir M, Melek M. Assessment of ventricular repolarization inhomogeneity in patients with mitral valve prolapse: value of T wave peak to end interval. Int J Clin Exp Med 2014;7:2173-8.

15. Karaagac K, Yontar OC, Tenekecioglu E, Vatansever F, Ozluk $\mathrm{OA}$, Tutuncu A, et al. Evaluation of Tp-Te interval and Tp-Te/ QTc ratio in patients with coronary artery ectasia. Int J Clin Exp Med 2014;7:2865-70.

16. Panikkath R, Reinier K, Uy-Evanado A, Teodorescu C, Hattenhauer J, Mariani R, et al. Prolonged Tpeak-to-tend interval on the resting ECG is associated with increased risk of sudden cardiac death. Circ Arrhythm Electrophysiol 2011;4:441-7. [CrossRef]

17. Sakagami J, Kataoka K, Sogame Y, Usui N, Kanemitsu D, Takada $\mathrm{R}$, et al. Increased QT dispersion in patients with alcoholic pancreatitis. Pancreas 2004;28:380-6. [CrossRef] 